\title{
RESEARCH
}

Open Access

\section{EGFR signaling confers resistance to BET inhibition in hepatocellular carcinoma through stabilizing oncogenic MYC}

Yalei Yin ${ }^{1}$, Mingju Sun ${ }^{1}$, Xi Zhan ${ }^{1,2}$, Changqing Wu', Pengyu Geng ${ }^{1}$, Xiaoyan Sun ${ }^{1,5,6,7,8}$, Yunsong $\mathrm{Wu}^{1,4}$, Shuijun Zhang ${ }^{5,6,7,8}$, Jianhua Qin ${ }^{1}$, Zhengping Zhuang ${ }^{3}$ and Yang Liu ${ }^{1,9^{*}}$

\begin{abstract}
Background: The bromodomain and extra-terminal domain (BET) inhibitor is a type of anti-tumor agent, currently being evaluated in phase I and II clinical trials for cancer therapy. It can decrease MYC expression levels and cause effective anti-tumor effects in diverse human cancers. However, its cytotoxic effect and related mechanisms of drug resistance are poorly understood in hepatocellular carcinomas (HCC). Here, we investigated the anti-tumor effects of BET inhibitor on HCC and the molecular mechanisms involved in its associated drug resistance.
\end{abstract}

Methods: We assessed the cytotoxicity of BET inhibitor on HCC cells compared with sorafenib by cell viability assay, metastasis assay and reproduced the anti-tumor effect in xenograft mouse model. In addition, the molecular mechanisms involved in drug resistance on JQ1-resistant HCC cells were revealed by western blotting, qRT-PCR, whole exome-sequencing and gene-editing technology. Finally, with specific inhibition of EGFR or ERK activity by interference RNAs or inhibitors, the efficacy of the synergistic treatment was investigated using cell viability assay, colony formation, apoptosis and xenograft mouse model.

Results: We found that JQ1, a commonly used BET bromo-domain inhibitor, offered a better anti-tumor response than sorafenib in MYC-positive HCC cells by inducing apoptosis in vitro and in vivo. Unlike sorafenib, JQ1 treatment significantly impaired mitochondrial respiration and glycolysis in HCC cells. Importantly, we revealed that MAPK activation by a previously undescribed activating mutation of EGFR-1645L, was critical for JQ1 sensitivity through stabilizing oncogenic MYC protein in JQ1-resistant HCC cells. Inhibition of either EGFR or ERK activity overcame the JQ1 resistance and significantly decreased MYC protein level in vitro and in vivo.

Conclusion: Since MYC amplification is frequently identified in HCC, co-occurring with EGFR amplification, our findings suggest that targeting EGFR signaling might be essential for JQ1 therapy in advanced HCC.

Keywords: Bromodomain, JQ1, MAPK pathway, MYC, EGFR mutation, Sorafenib

\footnotetext{
* Correspondence: yliuqq@dicp.ac.cn

This paper is dedicated to the 70th anniversary of the Dalian Institute of Chemical Physics, Chinese Academy of Sciences.

${ }^{1}$ CAS Key Laboratory of Separation Science for Analytical Chemistry, Scientific

Research Center for Translational Medicine, Dalian Institute of Chemical

Physics, Chinese Academy of Sciences, Dalian 116023, China

${ }^{9}$ University of Chinese Academy of Sciences, Beijing 100049, China

Full list of author information is available at the end of the article
}

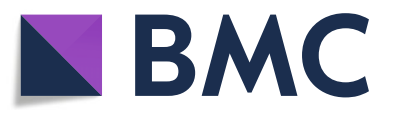

(c) The Author(s). 2019 Open Access This article is distributed under the terms of the Creative Commons Attribution 4.0 International License (http://creativecommons.org/licenses/by/4.0/), which permits unrestricted use, distribution, and reproduction in any medium, provided you give appropriate credit to the original author(s) and the source, provide a link to the Creative Commons license, and indicate if changes were made. The Creative Commons Public Domain Dedication waiver (http://creativecommons.org/publicdomain/zero/1.0/) applies to the data made available in this article, unless otherwise stated. 


\section{Background}

Sorafenib, a multiple kinase inhibitor, is currently the standard first-line drug approved by the Food and Drug Administration (FDA) for advanced hepatocellular carcinoma (HCC) [1-3]. Although sorafenib improves overall survival of HCC patients, the tumor response is generally poor [4-7]. Recently, lenvatinib and regorafinib, were approved as the first-line and second -line treatment for $\mathrm{HCC}$, respectively [8]. However, similarly to sorafenib, both of these two drugs inhibit tumor angiogenesis by interfering with multiple receptor tyrosine kinases (VEGFR, PDGFR), potentially leading to more severe intratumor hypoxia and drug resistance [9-11]. Nivolumab, a checkpoint-inhibitor was approved as second-line drug for the treatment of advanced HCC patients, the safety and efficacy are still being evaluated in clinic [12, 13]. Therefore, there is an urgent need to develop novel therapeutic drugs for HCC treatment.

Previous studies showed that high expression levels of MYC, a critical regulator of cell proliferation and metabolism [14-16], were observed in about $20 \%$ of HCC tumors and significantly correlated with tumorigenesis [17, 18]. Therefore, inhibition of MYC expression by small molecules has been shown to have great therapeutic potential for HCC treatment [19]. Bromodomain-containing protein 4 (BRD4) is a member of the bromodomain and extra-terminal (BET) family proteins, recruiting transcriptional regulatory proteins for binding to transcription start sites of genes, such as MYC, BCL2 and BCL6 genes [20, 21]. JQ1, a specific and competitive inhibitor of BRD4, can decrease MYC expression levels and induce apoptosis in diverse human cancers, including medulloblastoma, lung adenocarcinoma, glioblastoma, and myeloid leukemia [22-24]. Although JQ1 is still being evaluated in phase I and II clinical trials for the treatment of advanced malignancies [25], emerging evidence demonstrated that the anti-tumor effect of JQ1 was limited in tumor cells with high level of MYC protein $[26,27]$. It has been documented that PI3K/ $\mathrm{AKT} / \mathrm{mTOR}$ and MAPK/ERK activation may be involved in intrinsic resistance of JQ1 in tumor cells $[28,29]$. Furthermore, recent study showed that BRD4 could be considered as an attractive therapeutic target for HCC treatment [30]. However, since the sensitivity of this drug varies across a number of differential HCC cell lines, it is crucial to understand the molecular basis of JQ1 resistance in HCC cells with high level of MYC expression.

In this study, we sought to determine the anti-tumor effects of the BET inhibitor and the molecular mechanisms adopted by HCC cells against this drug. As a result, we demonstrated that the anti-tumor effects of JQ1 were more potent than those of sorafenib in MYC-positive HCC cell lines. In addition, whole exome-sequencing (WES) revealed a novel activating EGFR mutation-I645L, mediating JQ1 resistance by activating MAPK pathway and stabilizing MYC protein in HCC cells. Combined treatment of JQ1 with EGFR or ERK inhibitor significantly resulted in tumor growth inhibition in vitro and in vivo. Our findings suggest that combination of JQ1 with EGFR/MAPK inhibition may be an attractive therapeutic strategy in advanced HCC with EGFR activation.

\section{Materials and methods}

\section{Cell lines, plasmid transfection, viral infection}

The HCC cell lines Hep3B, HCCLM3(LM3), HuH7, HB611, HepG2, SMMC7721, MHCC97-L (97-L), MHCC97-H (97-H), PLC/PRF/5 and BEL-7402 were purchased from the ATCC and maintained in Dulbecco's modified Eagle's medium or RPMI-1640 medium supplemented with $10 \%$ fetal bovine serum at $37^{\circ} \mathrm{C}$ in a humidified atmosphere with $5 \% \quad \mathrm{CO}_{2}$. The EGFR-WT and EGFR-I645L cDNAs were obtained from 97-L and 97-H cells following RNA isolation and subsequent reverse transcription PCR (Takara, Japan). cDNAs of wild type and mutanted EGFR were cloned into pCDH-EF1-coGFP-puro lentiviral vector (CD513B1, SBI Inc., Mountain View, CA, USA) using $\mathrm{XbaI}$ and NheI restriction sites, respectively. Lentivirus based shRNA against EGFR was purchased from Origene Inc. (TL320326, Rockville, MD, USA). For plasmid transfection, Lipofectamine 2000 was used according to the standard protocol. Plasmids were co-transfected with the packaging plasmid (TR30022, Origene, Rockville, MD, USA) into $293 \mathrm{~T}$ cells to generate the viral supernatant, then the viral supernatant infected cells. Stable HCC cell lines were established in culturing with $4 \mu \mathrm{g} / \mathrm{ml}$ puromycin (Thermo-Fisher, MA, USA).

\section{Reagents}

JQ1, Sorafenib (Sora), AZD6244 (AZD), SCH772984 (SCH) and Erlotinib (ERL) were purchased from Selleck (Houston, TX, USA) and diluted in DMSO. Cycloheximide (CHX) was purchased from MCE (Shanghai, China). Antibodies against BRD4 (\#13440), MYC (\#5605), p-MYC-Ser62 (\#13748), PARP (\#9542), cleaved-PARP (\#5625), cleaved-Caspase3 (\#9664), ERK1/2 (\#4695), p-ERK1/2(T202/Y204) (\#4370), AKT (\#2920), p-AKT(S473) (\#4060), EGFR (\#4267), p-EGFR(Tyr1068) (\#3777), cleaved-Caspase3 (\#9664) and GAPDH (\#5174) were purchased from Cell signaling Technology (CST, Boston, USA). Antibodies against p-MYCThr58 (\#28842), Ras (\#52939) and p-MYC-Ser62 (\#185656) 
for immunohistochemistry were purchased from abcam (Cambridge, UK). The $\beta$-Actin antibody was purchased from Santa Cruz Inc.(Dallas, TX, USA).

\section{Cell viability assay}

For the cell viability assay, $5.0 \times 10^{3}$ cells per well were plated in 96-well plates and cultured in a $5 \% \mathrm{CO}_{2}$ incubator. After $24 \mathrm{~h}$, compounds were added in serial dilutions. $48 \mathrm{~h}$ later, cell viability was assessed using the Cell Titer-Glo ${ }^{\circ}$ Luminescent Cell Viability Assay (Promega, Madison, WI, USA) following the protocols provided by the manufacturers.

\section{Extracellular acidification rate (ECAR)}

ECAR assay was determined by Seahorse XF96e analyzer (Seahorse Bioscience, USA) according to the manufacturer's instructions. Briefly, $1.0 \times 10^{4}$ cells were plated per well in a 24-well XF cell culture microplate. ECAR was measured in XF base medium containing $4 \mathrm{mM}$ glutamine ( $\mathrm{pH} 7.35$ ) following sequential additions of glucose $(10 \mathrm{mM})$, oligomycin (1 $\mathrm{mM})$ and 2-DG $(50 \mathrm{mM})$. Data were analyzed by a Seahorse XF Glycolysis Stress Test Report Generator.

\section{Colony formation assay}

For colony formation assay, $1.0 \times 10^{4}$ cells were plated into 6-well dishes in $2 \mathrm{ml}$ of medium containing $0.3 \%$ agarose, overlaid with $2 \mathrm{ml}$ of $0.5 \%$ agarose. DMEM was added on top, and maintained in a humidified atmosphere with $5 \% \mathrm{CO}_{2}$ at $37^{\circ} \mathrm{C}$ for 6 weeks. Then colonies were stained with crystal violet, and the number of colonies was counted.

\section{Quantitative real-time PCR}

Total RNA was isolated from cell pellets with the Trizol ${ }^{\circ}$ Reagent (Takara, Japan) and reverse-transcribed into cDNA using SYBR premix EX Taq (Takara, Japan) and specific primers. Gene expression level was assessed by qRT-PCR using the CFX96 Touch $^{\text {Tu }}$ Real-Time PCR Detection System according to the manufacturer's instructions. GAPDH was used as the loading control. MYC primer sequences were: F:5'-CGTCCTCGGATTCTCTGCTCTC-3'， R: 5'-GCGC TGCGTAGTTGTGCTGAT-3'. GAPDH primer sequences were: F: 5'CTTCGCTCTCTGCTCCTCCTGTTCG-3', R: 5'ACCAGGCGCCCAATACGACCAAAT-3'.

\section{Annexin V/ Propidium iodide (PI) double-staining assay} Annexin V-FITC apoptosis detection kit (BD biosciences, USA) was used to assess the apoptosis. Annexin $\mathrm{V}$ and PI staining was performed according to the manufacturer's protocol. Briefly, $1.0 \times 10^{5}$ cells in $200 \mu \mathrm{L} 1 \times$ Binding Buffer were incubated with $10 \mu \mathrm{L}$ FITC Annexin V and $5 \mu \mathrm{L}$ PI for $30 \mathrm{~min}$ at room temperature. Stained cells were detected using a FACScan flow cytometer (BD biosciences) and data was collected using FlowJo software.

\section{Western blotting}

Immunoblotting was carried out using standard protocol. Briefly, cells were lysed in ice-cold $1 \times$ RIPA lysis buffer, and protein concentrations were determined by bicinchoninic acid (BCA, Tiangen, China). Cell lysates were loaded on precast 4 to $10 \%$ NuPAGE Novex 4 to $12 \%$ Bis-Tris Protein Gels (Life technologies, Carlsbad, CA). Then the proteins were transferred onto PVDF membranes, subsequently blocked and incubated with primary antibodies overnight at $4{ }^{\circ} \mathrm{C}$. After washing, the membranes were probed with HRP conjugated rabbit-anti-goat secondary antibodies (1:4000) for $2 \mathrm{~h}$ at room temperature. Targeted proteins were visualized using enhanced chemiluminescence (Thermo-Fisher, MA, USA) on Hyperfilm (GE Healthcare, MA, USA).

\section{Xenograft mice model}

All animal procedures were performed in accordance with the NIH Guide on the Care and Use of Laboratory Animals and approved by the Institutional Animal Care and Use Committee of the Dalian Medical University. $5 \times 10^{6}$ Cells suspended in a mixture of Matrigel and $1 \times$ PBS were implanted subcutaneously into the flanks of 5-week-old CB17/SCID mice. For the experiment to determine JQ1 and sorafenib in vivo cytotoxicty, we injected BEL-7402 and 97-L cells into immunodeficient nude mice to generate $\mathrm{HCC}$ xenograft tumors, respectively. When tumors nodules had been formed and reached a volume of $100 \mathrm{~mm}^{3}$, the xenograft mice were divided into three groups and treated with vehicle, JQ1 or sorafenib respectively at $50 \mathrm{mg} / \mathrm{kg}$ every 2 days. For the experiment to determine the anti-tumor effect of combined treatment in vivo, 97-H cells were implanted into immunodeficient nude mice for subcutaneous xenograft tumor formation. When tumors nodules reached a volume of 100 $\mathrm{mm}^{3}$, mice were divided into four groups and treated with Vehicle, JQ1 $(50 \mathrm{mg} / \mathrm{kg})$, ERL $(50 \mathrm{mg} / \mathrm{kg})$ or a combination of both drugs every 2 days. Tumors were measured with calipers and tumor volume $\mathrm{V}$ was calculated using the equation: $V=\pi / 6 \times$ length $\times$ width $^{2}$.

\section{Immunohistochemistry}

Tumor xenografts were embedded in Tissue-Tek OCT (Sakura, USA) for frozen liver sections (thickness $10 \mu \mathrm{m}$ ) with a standard procedure. For immunohistochemistry (IHC), the slides were fixed with $4 \%$ paraformaldehyde. $3 \%$ peroxidase and $20 \%$ fetal 


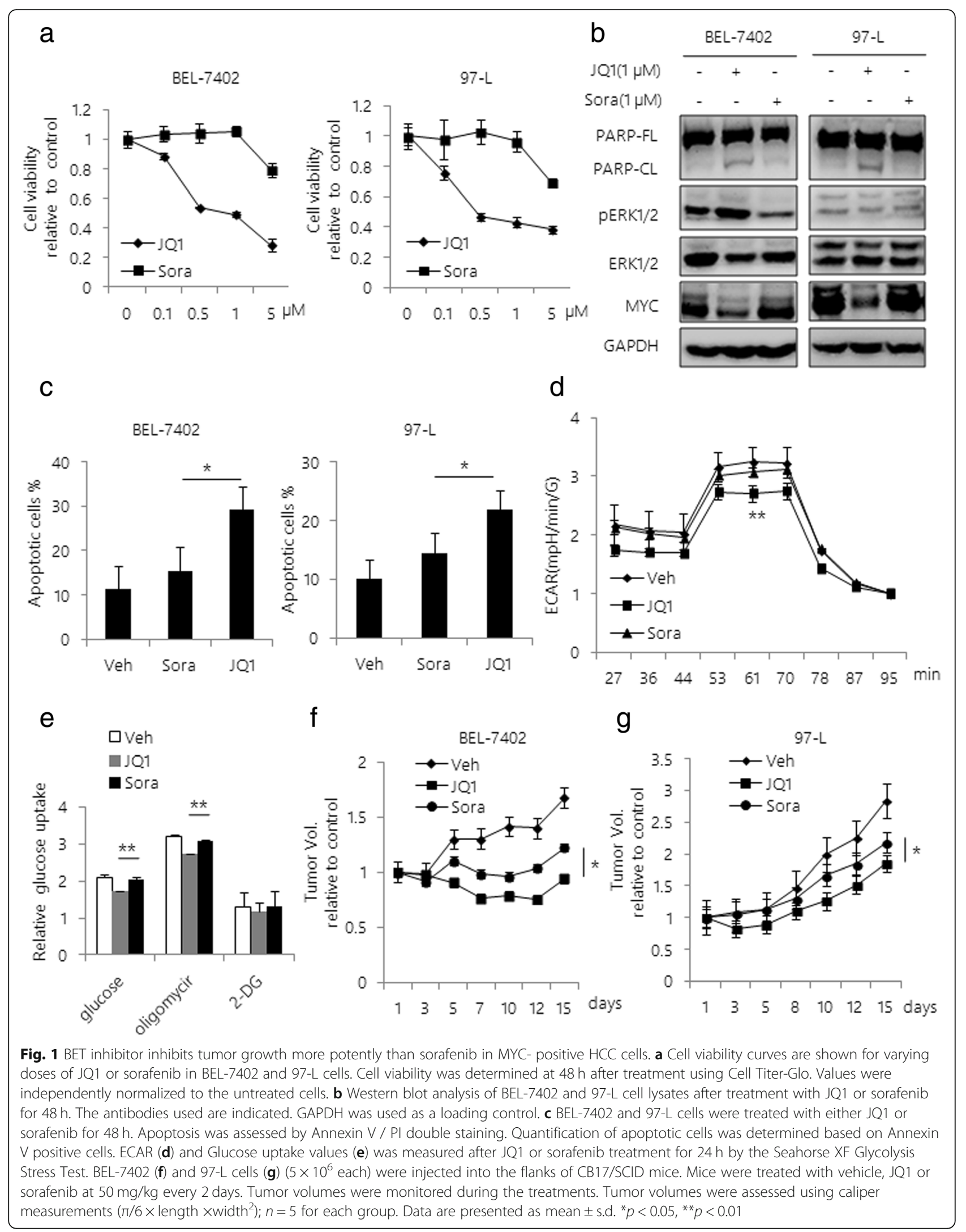




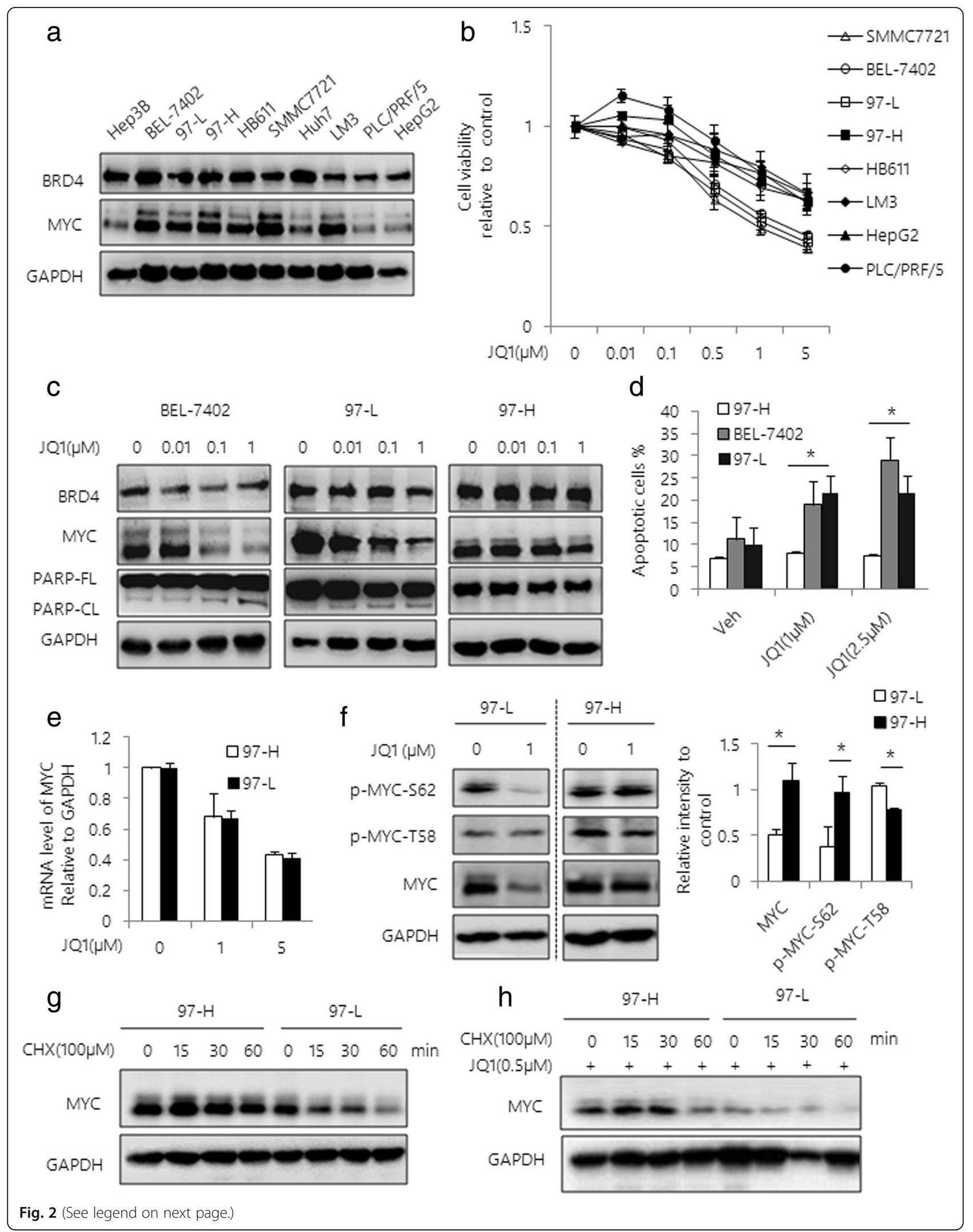


(See figure on previous page.)

Fig. 2 JQ1 selectively inhibits HCC cell tumor growth. a Western blot analysis of BRD4 and MYC expression in 10 HCC cell lines. GAPDH was Used as a loading control. b Cell viability curves are shown for serial dilutions of JQ1 in $8 \mathrm{HCC}$ cell lines. Cell viability was determined at $48 \mathrm{~h}$ after treatment using Cell Titer-Glo. c Western blot analysis of BEL-7402, 97-L and 97-H cells treated with serial dilutions of JQ1 for $48 \mathrm{~h}$. Total lysates were subjected to the indicated antibodies. d BEL-7402, 97-L and 97-H cells were treated with varying dose of JQ1 for $48 \mathrm{~h}$. Apoptosis was assessed by Annexin V / PI double staining. Quantification of apoptotic cells was determined based on Annexin V positive cells. e 97-L and 97-H cells were treated with JQ1 for $48 \mathrm{~h}$. MYC mRNA expression level was analyzed by qRT-PCR. $\mathbf{f}$ Western blot analysis of 97-L and 97-H cells treated with 1 MM JQ1 for $48 \mathrm{~h}$. Expressions of MYC, p-MYC-Ser62 and p-MYC-Thr58 were examined. Band intensities were quantified by Image J software and graphed at the right side. GAPDH was used as a loading control. Western blot analysis of 97-L and 97-H cells incubated with $100 \mu \mathrm{M}$ CHX in the absence $(\mathbf{g})$ or presence $(\mathbf{h})$ of JQ1. Data are presented as mean \pm s.d. ${ }^{*} p<0.05$

bovine serum were diluted in $1 \times$ PBS to block endogenous peroxide activity and nonspecific binding. The slides were incubated with primary antibodies overnight at $4{ }^{\circ} \mathrm{C}$ followed by biotin-streptavidin HRP detection systems (ZSGB-BIO, China). Hematoxylin dye was used as counter stain. The slides were examined with an Olympus BX61 microscope with cellSens Standard Software Version 1.6 (Olympus Corporation, Tokyo, Japan).

\section{Statistical analysis}

Statistical analyses were performed using Microsoft Excel software. Results were representative of three independent experiments with three replicates. Student's t-tests were used to analyze the statistical significance of differences between two groups. $P$-value of $<0.05$ was considered statistically significant in all cases and indicated by one asterisk. Error bars shown in the figures represent s.d.

\section{Results}

BET inhibitor inhibits tumor growth more effectively than sorafenib in MYC-positive HCC cells

First, we investigated whether MYC amplification is correlated with clinical characteristics in different public transcriptomic datasets [31]. We found that high levels of MYC mRNA are associated with high risk for tumor pathogenesis and poor prognosis in HCC (Additional file 1: Figure S1a and b). Next, to evaluate the anti-tumor potential of BET inhibition on HCC cells, we investigated the cytotoxicity of JQ1 on HCC cells in comparison with sorafenib. The results showed a sharp reduction of cell viability in response to JQ1 in BEL-7402 and 97-L cell lines, while sorafenib treatment caused a mild growth inhibition (Fig. 1a). As expected, JQ1 treatment significantly decreased levels of MYC in both cell lines and induced cellular apoptosis supported by increase in PARP cleavage and Annexin V+population, while sorafenib treatment did not induce cellular apoptosis in these cells (Fig. 1b, c and Additional file 2: Figure S2).
Since the increased dependency of anaerobic glycolysis is critical for HCC pathogenesis [32], we examined the effect of JQ1 and sorafenib on cellular glycolysis in HCC. We found that JQ1 significantly impaired mitochondrial respiration by attenuating glycolytic metabolism in HCC, supported by decreased Extracellular Cidification Rate (ECAR) comparing to sorafenib-treated cells (Fig. 1d and e). Next, to determine if JQ1 could reproduce the anti-tumor effect in HCC mice model, we injected BEL-7402 and 97-L cell lines subcutaneously into immunodeficient nude mice to generate HCC xenograft tumors. We found that JQ1 was able to reduce in vivo tumor growth more efficiently than sorafenib (Fig. 1f and g). The average tumor volumes were significantly smaller in JQ1 treatment group than sorafenib treated mice group (Additional file 3: Figure S3a). Furthermore, in consistent with our in vitro results, both decreased levels of MYC and induction of apoptosis were observed in JQ1 treated tumor xenografts by IHC staining (Additional file 3: Figure S3b and c). In contrast, we did not observe significant apoptosis in sorafenib treated mice group.

\section{JQ1 selectively inhibits HCC cell tumor growth in vitro} To further investigate the anti-tumor effect of JQ1 in a variety of $\mathrm{HCC}$ cells, we assessed the expression levels of BRD4 and MYC in ten HCC cell lines. The relatively high levels of $\mathrm{MYC}$ and BRD4 expression were identified in 6 out of 10 cell lines, including BEL-7402, 97-L, 97-H, HB611, SMMC-7721 and HCCLM3 cells (Fig. 2a). Furthermore, eight HCC cell lines were tested for the cytotoxicity of JQ1. We found that the cytotoxic effect of JQ1 was positively correlated with MYC status (Fig. 2b). Notably, JQ1 significantly induced cytotoxicity in a dose dependent manner on HCC cells with relatively high MYC levels, including SMMC-7721, BEL-7402 and 97-L cells (Fig. 2b). The on-target effect of JQ1 was confirmed by western blot analysis, showing the dose-dependent reduction of MYC levels (Fig. 2c). In addition, induction of cellular apoptosis by JQ1 was observed by PARP cleavage, and further supported by Annexin V / PI double staining (Fig. 2d and Additional file 4: Figure 


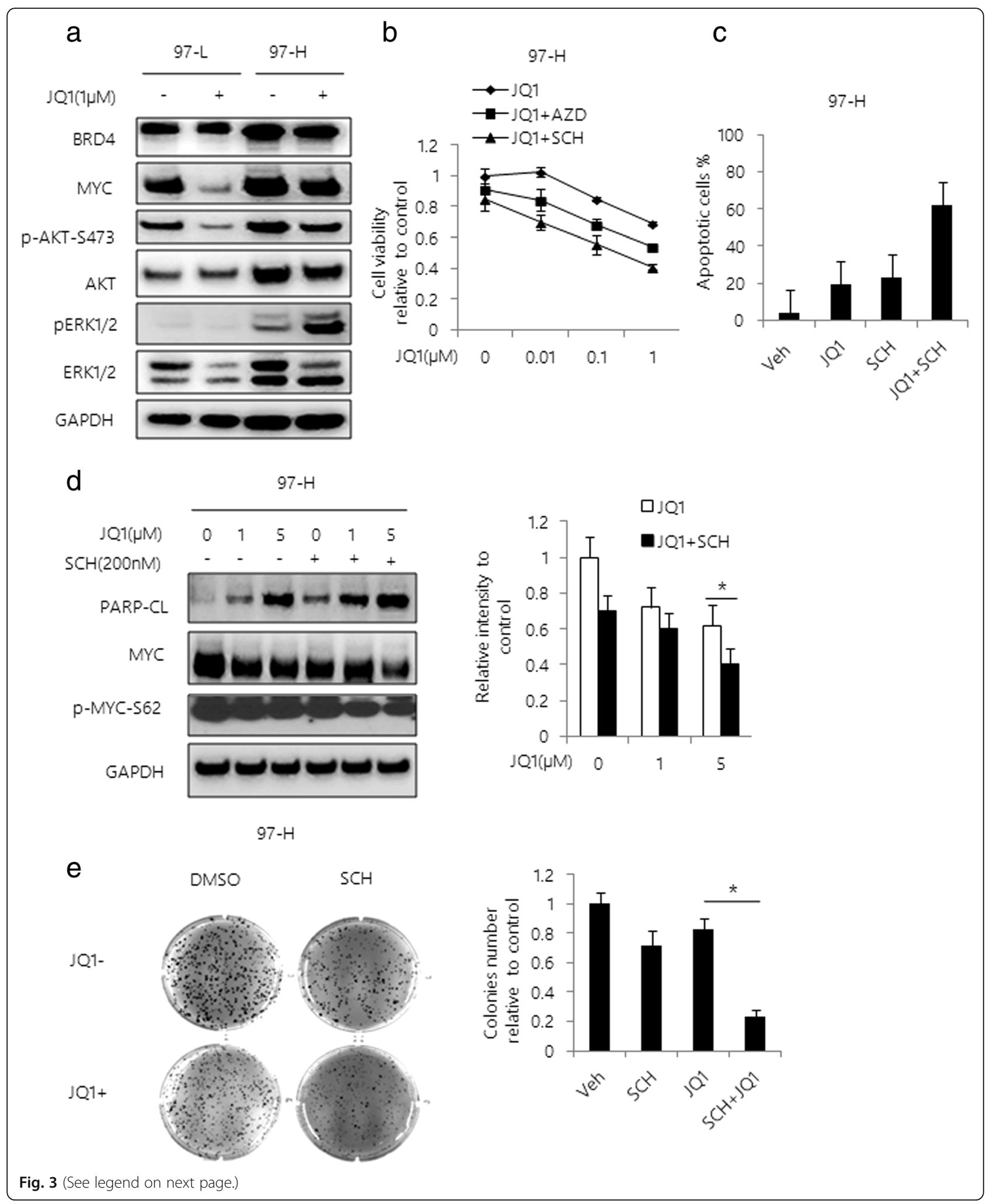




\section{(See figure on previous page.)}

Fig. 3 Activation of MAPK pathway mediates JQ1 resistance in HCC. a Western blot analysis of 97-L and 97-H cells treated with $1 \mu \mathrm{M} J \mathrm{~J} 1 \mathrm{for} 48 \mathrm{~h}$. Total lysates were subjected to the indicated antibodies. b Cell viability curves are shown for varying doses of JQ1 with or without 200 nM SCH or AZD in 97-H cells. Cell viability was determined using Cell Titer-Glo at $48 \mathrm{~h}$ after incubation. c 97-H cells were treated with vehicle, JQ1, SCH or the combination for $48 \mathrm{~h}$. Apoptosis was assessed and quantified by Annexin V / PI double staining. Quantification of apoptotic cells was determined based on Annexin V positive cells. $\mathbf{d}$ Western blot analysis of 97-H cells treated with varying doses of JQ1 with or without $200 \mathrm{nM}$ $\mathrm{SCH}$ for $48 \mathrm{~h}$. Total lysates were subjectd to the indicated antibodies. Band intensities for MYC were quantified by Image J software and graphed at the right side. e Colony formation assays were performed in 6-well plates. 97-H cells were treated with vehicle, 1 HM JQ1, 200 nM SCH or the combination. After 6 weeks of incubation, colonies were stained with crystal violet and the number of colonies per well was determined and graphed at the right side. Data are presented as mean \pm s.d. ${ }^{*} p<0.05$

S4). While we found that JQ1 failed in decreasing the cell activity on HCC cells with lower MYC level, such as HepG2 and PLC/PRF/5 cells. Interestingly, we observed differential cytotoxic response to JQ1 in $97-\mathrm{L}$ and $97-\mathrm{H}$ cells, which are originated from the same HCC tumors [33]. Unlike 97-L, 97-H cells were relatively insensitive to JQ1 and were characterized by sustained MYC expression regardless of JQ1 treatment. To determine the possible mechanisms involved in the cellular resistance to JQ1, we assessed the mRNA level of MYC in JQ1-treated cells by qRT-PCR, showing similar reduction of MYC mRNA levels in both of these two cell lines (Fig. 2e). These results led us to examine an involvement of posttranscriptional regulation of MYC protein in JQ1-treated 97-H cells. Indeed, we observed sustained phosphorylation of MYC at Ser62 site (S62), which is crucial for preventing oncogenic MYC from proteasomal degradation [34]. In contrast, the level of phosphorylation of MYC at Thr58 (T58), which is critical for degradation of MYC protein, was significantly decreased by JQ1 in 97-H cells. While there was no significant change of level of p-MYC-Thr58 in 97-L cells (Fig. 2f). To further assess the difference of MYC stability between these two cells, we treated the cells with cycloheximide ( $\mathrm{CHX}$ ), a molecule to block protein synthesis. As expected, we found that $\mathrm{CHX}$ significantly reduced MYC protein level in 97-L cells, but not in 97-H cells with or without treatment of JQ1, suggesting the enhanced protein half-life of MYC in $97-\mathrm{H}$ cells (Fig. $2 \mathrm{~g}$ and $h$ ).

\section{MAPK activation is critical for JQ1 sensitivity}

As shown above, 97- $\mathrm{H}$ cells were relatively resistant to JQ1 compared to 97-L cells. Since 97-L and 97-H cell lines have similar origins, we believed that these two cell lines could be good models to study the mechanism of acquired cellular resistance to JQ1. To investigate the key factors in preventing MYC from proteasomal degradation in JQ1-treated $97-\mathrm{H}$ cells, we evaluated the effects of JQ1 on PI3K/AKT and MAPK/ERK signaling pathways, which have been shown to be associated with JQ1 resistance and MYC posttranscriptional regulation [26]. No significant difference in AKT pathway activity was observed between 97-L and 97-H cells after JQ1 treatment. In contrast, the phosphorylation level of ERK was significantly up-regulated in 97-H cells compared with 97-L cells, with or without JQ1 treatment (Fig. 3a). To determine if inhibition of ERK activity could affect the response of $97-\mathrm{H}$ cells to JQ1, we treated these cells with different concentrations of JQ1 in combination with specific ERK inhibitors, AZD6244 or SCH772984 $(\mathrm{SCH})$ respectively. We found that the combination treatment significantly reduced cell viability and growth in 97-H cells (Fig. 3b). In addition, the Annexin V/PI dual staining also showed that cellular apoptosis was induced by the combined treatment (Fig. 3c and Additional file 5: Figure S5). Furthermore, a remarkable reduction in MYC protein expression and the phosphorylation of MYC protein levels were observed in the combined treatment of JQ1 and $\mathrm{SCH}$ (Fig. 3d). The combined treatment also significantly abolished the colony forming ability of $97-\mathrm{H}$ cells, comparing to JQ1 alone (Fig. 3e). Taken together, these results demonstrated that MAPK pathway may be critical for MYC stability and JQ1 resistance.

\section{Acquired EGFR mutation stabilizes MYC protein in the presence of JQ1}

To investigate the discrepancy of upstream mechanisms regulating the MAPK pathway in JQ1 insensitive HCC cells, we performed the WES analysis in 97-H and 97-L cells. We identified a previously undescribed EGFR-I645L mutation in $97-\mathrm{H}$ cells. The presence of this mutation was further confirmed by Sanger sequencing (Fig. 4a). Next, to determine if EGFR-I645L is potentially associated with JQ1 resistance in $97-\mathrm{H}$ cell line, we first examined the effects of EGFR-I645L on activation of EGFR and MEK/ ERK pathway. As expected, we found that both p-EGFR and p-ERK levels were much higher in 97-H cells than that of 97-L cells, regardless of Extracellular Growth Factor (EGF) status (Fig. 4b). To further characterize the 


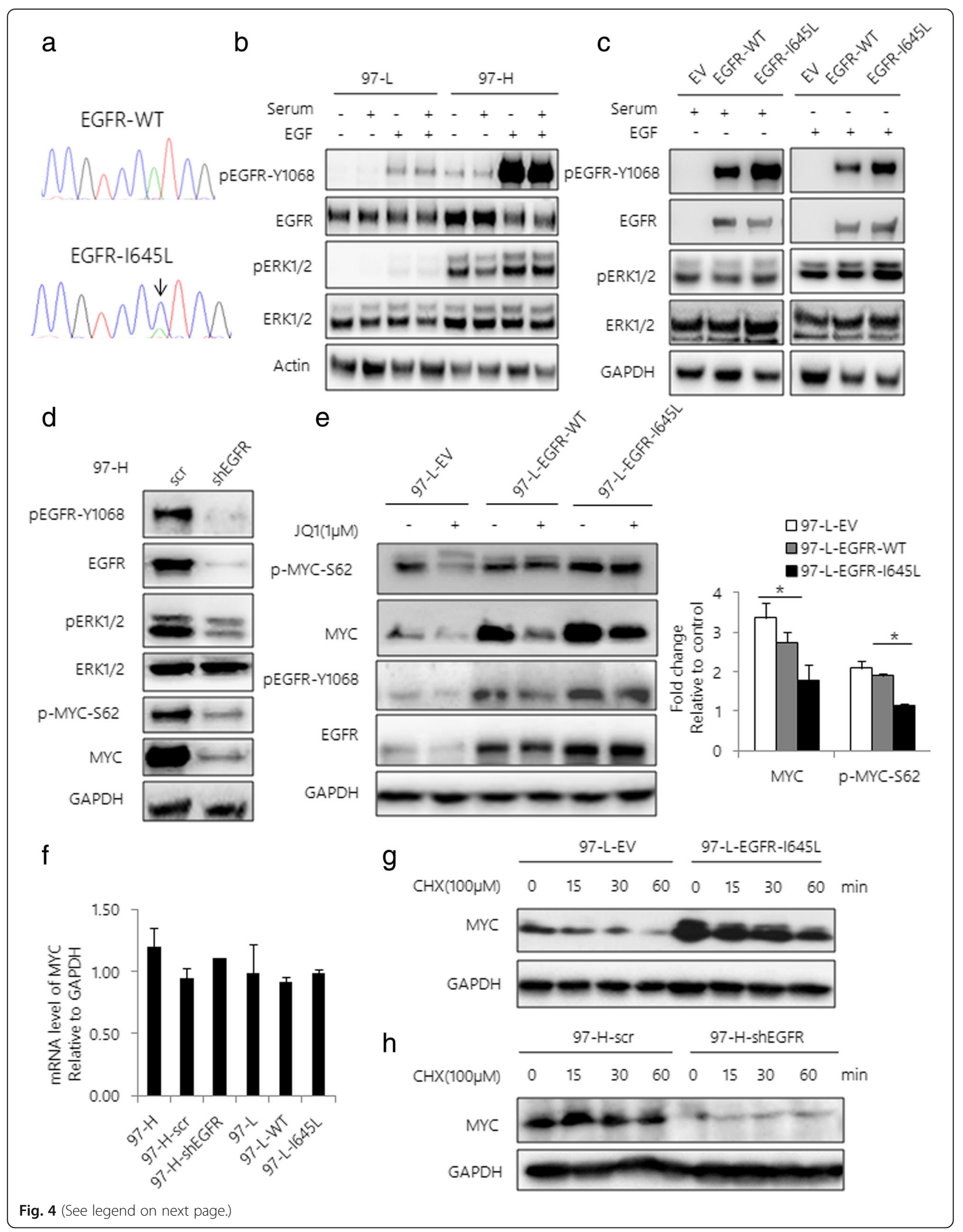


(See figure on previous page.)

Fig. 4 Acquired EGFR mutation confers JQ1 resistance by stabilizing MYC. a Diagram of the EGFR-1645L mutation in 97-H cells identified by Sanger sequencing of EGFR mRNA. b Western blot analysis of $97-\mathrm{L}$ and $97-\mathrm{H}$ cells with or without EGF stimulation after serum starvation $\pm 10 \%$ fetal bovine serum (FBS) for 30 min. Total lysates were subjected to the indicated antibodies. c Western blot analysis of EGFR-WT or EGFR-1645Lexpressing 293 T cells lysates after serum starvation or EGF stimulation. Total lysates were subjected to the indicated antibodies. EV is the empty vector-transfected $293 \mathrm{~T}$ cells. d Western blot analysis of $97-\mathrm{H}$ cells transfected with scrambled shRNA or shEGFR. Total lysates were subjected to the indicated antibodies. e Western blot analysis of EV, EGFR-WT or EGFR-1645L-expressing 97-L cell lysates after JQ1 treatment. Total lysates were subjected to the indicated antibodies. Band intensities were quantified by Image J software and graphed at the right side. $\mathbf{f}$ MYC mRNA expression level in 97-H cells transfected with scrambled shRNA or shEGFR and EGFR-WT or EGFR-1645L-expressing 97-L cells were assessed using qRT-PCR. $\mathbf{g}$ Western blot analysis of 97-L-EV and 97-L-EGFR-1645L cells incubated with $100 \mu$ M CHX. $\mathbf{h}$ Western blot analysis of 97-H-scr and 97-HshEGFR cells incubated with $100 \mu \mathrm{M}$ CHX. Data are presented as mean \pm s.d. ${ }^{*} p<0.05$

function of this mutation, we over-expressed both EGFR-WT and EGFR-I645L mutant in 293 T cells respectively. In agreement with the results above, expression of EGFR-I645L mutant significantly increased the level of both p-EGFR-Y1068 and p-ERK compared to EGFR-WT under either serum or EGF stimulation, suggesting that EGFR-I645L is an activating mutation (Fig. 4c). To further determine if EGFR-I645L mutant contributes to JQ1 resistance through activating MAPK pathway, we knocked down expression of EGFR in 97-H cells, showing that inhibition of EGFR activity significantly reduced the levels of p-EGFR and p-ERK, subsequently decreased the levels of both MYC and p-MYC-Ser62 (Fig. 4d). In contrast, overexpression of EGFR-I645L mutant increased p-MYC-Ser62 and MYC levels in 97-L cells compared to those expressing EGFR-WT, in the absence or presence of JQ1 (Fig. 4e). Moreover, neither knockdown nor overexpression of EGFR affected MYC mRNA levels (Fig. 4f). Furthermore, expression of EGFR-I645L mutant enhanced the half-life of MYC protein upon the treatment of $\mathrm{CHX}$ (Fig. 4g). In contrast, knockdown of EGFR dramatically decreased MYC protein level in the presence of CHX (Fig. 4h). These results suggest that EGFR/ERK activation is critical for stabilizing MYC protein.

\section{EGFR inhibition sensitizes HCC cells to JQ1}

Next, to examine if inhibition of EGFR activity could overcome JQ1 resistance in HCC cells, we knocked down EGFR by lentiviral vector-based shRNA infection. We found that loss of EGFR expression significantly enhanced the JQ1-induced growth inhibition in 97-H cells (Fig. 5a). Moreover, MYC protein levels markedly decreased with induction of apoptosis following the treatment with JQ1 in absence of EGFR expression (Additional file 6: Figure S6a). The colony formation assay also confirmed that EGFR inhibition reduced the number of colonies of $97-\mathrm{H}$ cells with JQ1 treatment (Fig. 5b). On the other hand, the expression of EGFR-I645L mutant in 97-L cells attenuated the anti-proliferative effects of JQ1, compared to EGFR-WT (Fig. 5c). To evaluate the synergistic effects, we treated 97-H cells with Erlotinib (ERL), an specific EGFR inhibitor, in combination with varying doses of JQ1. The results showed that the combined treatment of ERL with JQ1 significantly inhibited 97-H cell growth compared to either ERL or JQ1 alone (Fig. 5d). Moreover, the combination of ERL with JQ1 significantly induced the apoptosis of $97-\mathrm{H}$ cells in a dose-dependent manner, which was supported by increased Annexin $\mathrm{V}+$ population and PARP cleavage (Fig. 5e, Additional file 6: Figure S6b and $6 \mathrm{c}$ ). In addition, levels of MYC and p-MYC-Ser62 were effectively inhibited by combination of JQ1 with either ERL or SCH (Fig. 5f). On-target effect of combination treatment was also confirmed by western blot analysis of level of p-ERK and p-EGFR (Fig. 5f). Furthermore, the combined treatment of JQ1 with ERL almost completely abolished the colony forming ability of $97-\mathrm{H}$ cells, while the single drug treatments had limited inhibitory effects (Fig. $5 \mathrm{~g}$ ).

\section{Combination of JQ1 with EGFR inhibitor effectively reduces JQ1 resistant HCC tumor growth in vivo}

Next, we investigated the efficacy of the combination of JQ1 and EGFR inhibitor in inhibiting HCC tumor growth in vivo. In agreement with our in vitro results, we found that either JQ1 or ERL alone resulted in a modest inhibition of tumor growth. However, a significantly greater anti-tumor effect was observed in the group with combination treatment (Fig. 6a). Average tumor volumes were significantly smaller in mice subjected to the combined treatment compared to either agent alone (Fig. 6b). Consistently, we found that the combined treatment significantly decreased the levels of MYC and p-MYC-Ser62, compared to the JQ1 alone treatment (Fig. $6 \mathrm{c}$ and d). Furthermore, induction of apoptosis was also observed in the 


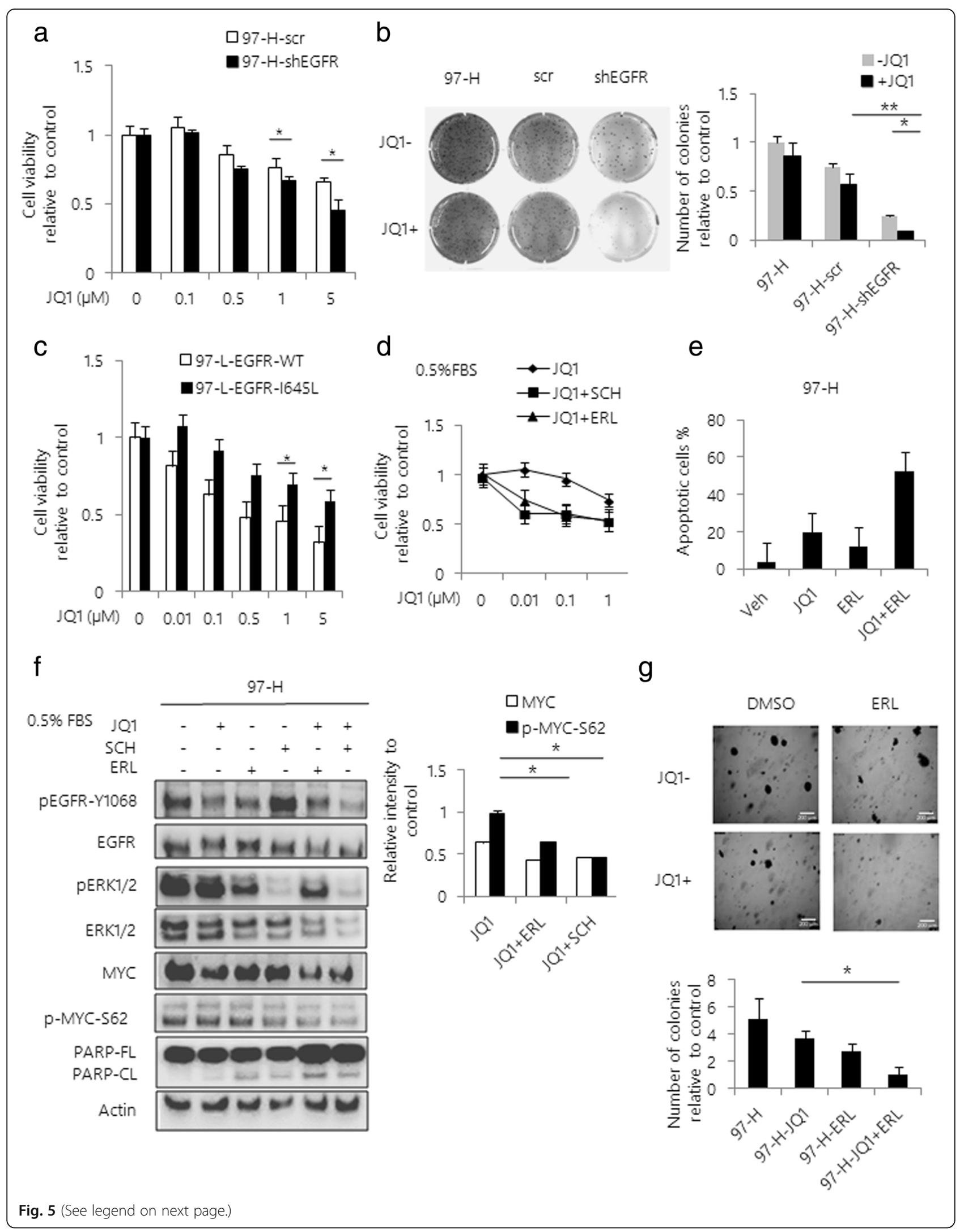


(See figure on previous page.)

Fig. 5 EGFR inhibition sensitizes HCC cells to JQ1. a Cell viability curves are shown for varying doses of JQ1 in 97-H cells transfected with scrambled shRNA or shEGFR. Cell viability was determined at $48 \mathrm{~h}$ after treatment using Cell Titer-Glo. b Colony formation assays were performed in 6-well plates. 97-H cells transfected with scrambled shRNA or shEGFR were treated with $1 \mu \mathrm{M}$ JQ1 for 6 weeks. After 6 weeks of incubation, colonies were stained with crystal violet and the number of colonies per well was determined and graphed at the right side. c Cell viability curves are shown for varying doses of JQ1 in EGFR-WT or EGFR-1645L expressing 97-L cells. Cell viability was determined at $48 \mathrm{~h}$ after treatment using Cell Titer-Glo. d Cell viability curves are shown for varying doses of JQ1 with or without a fixed dose of SCH or ERL in 97-H cells. e $97-\mathrm{H}$ cells were treated with vehicle, JQ1, ERL or the combination for 48 h. Apoptosis was assessed and quantified by Annexin V/PI double staining. Quantification of apoptotic cells was determined based on Annexin $V$ positive cells. $\mathbf{f}$ Western blot analysis of $97-\mathrm{H}$ cells treated with $1 \mu \mathrm{M}$ JQ1, $200 \mathrm{nM} \mathrm{SCH}, 1 \mu \mathrm{M}$ ERL or the combination of compounds for $48 \mathrm{~h}$. Total lysates were subjected to the indicated antibodies. Band intensities were quantified by Image J software and graphed at the right side. $\mathbf{g}$ Colony formation assays were performed in 6-well plates. 97-H cells were treated with vehicle, $1 \mu \mathrm{M}$ JQ1, $1 \mu \mathrm{M}$ ERL or the combination of two drugs. After 6 weeks of incubation, colonies were stained with crystal violet and the number of colonies per well was determined and graphed at following position Data are presented as mean \pm s.d. ${ }^{*} p<0.05,{ }^{* *} p<0.01$

combined treatment mice group, supported by increased level of TUNEL positive cells (Fig. 6d).

\section{Discussion}

MYC is a master oncogenic driver, regulating transcriptional programs to influence cell proliferation and metabolism in a variety of human cancers [35]. JQ1 is a specific and competitive inhibitor of BRD4, currently being evaluated in phase I and II clinical trials for advanced malignancies [36]. Since sorafenib is the first-line drug approved by FDA, we compared the anti-tumor effect of JQ1 with sorafenib, showing that JQ1 had more promising anti-tumor effects than sorafenib in HCC cells expressing relatively high level of MYC in vitro and in vivo. This result could be possibly interpreted by the high dependency of HCC tumor on glycolysis for energy production and MYC regulation of LDHA and PKM2, which play key roles in driving anaerobic glycolysis [32, 37]. Indeed, we showed that JQ1 more efficiently inhibited mitochondrial glycolysis than sorafenib.

Despite JQ1-induced growth inhibition of MYCpositive $\mathrm{HCC}$ cell lines, the responses varied significantly in these cells. Interestingly, we observed that JQ1 more effectively decreased MYC protein levels and induced cellular apoptosis in 97-L cells, comparing to $97-\mathrm{H}$ cells. Since these two cell lines were uniformly originated, we inferred that $97-\mathrm{H}$ cells might be a representative model to investigate the molecular basis of JQ1 resistance in HCC. Notably, our qRT-PCR results showed that JQ1 impaired the BRD4-mediated transcription activation of MYC in both $97-\mathrm{H}$ cells and 97-L cells, suggesting that a mechanism of posttranscriptional modification in stabilizing MYC protein in $97-\mathrm{H}$ cells. Indeed, continious phosphorylation of MYC Ser62 was observed in 97-H cells regardless of JQ1 treatment. This was also supported by the enhanced half-life of MYC in the presence of $\mathrm{CHX}$ in these cells, suggesting that sustained MYC activity might be critical for JQ1 sensitivity in $97-\mathrm{H}$ cells. Since MAPK signal is associated with JQ1 responses in tumors, we then examined the MAPK activation in response to JQ1 in $97-\mathrm{H}$ cells [29]. As expected. JQ1 treatment significantly activated p-ERK in 97-H cells. In addition, inhibition of ERK by a specific ERK inhibitor $\mathrm{SCH}$ overcame the JQ1 resistance and decreased MYC protein level, suggesting that MAPK pathway might be responsible for stability of MYC protein in HCC cells. This is consistent with previously study that ERK can directly interact with phosphorylated FBW7 to regulate the ubiquitination of FBW7 itself, subsequently preventing MYC degradation [34]. Therefore, it is essential to understand the mechanism of acquired MAPK activation in $97-\mathrm{H}$ cells.

The elevated ERK activity could result from the constitutive activation of upstream molecules, including EGFR and FGFR families, cytokines and hormones [26, 27]. Indeed, our WES results identified a previously undescribed EGFR-I645L mutation in 97-H cell line. Notably, we demonstrated that EGFR-I645L is an activating mutation, supported by the constitutive expression of p-EGFR-Y1068 and p-ERK1/2 regardless of serum or EGF stimulation. More importantly, in combination with the ERK inhibition, blockade of EGFR activity by either RNA interference or EGFR inhibitor effectively decreased the level of p-MYC-Ser62 and reduced cell growth by inducing apoptosis in $97-\mathrm{H}$ cells. In contrast, over-expression of EGFRI645L mutant remarkably increased the level of p-MY C-Ser62 in 97-L cells compared to the EGFR-WT. Furthermore, combination of JQ1 with EGFR inhibitor significantly reduced $97-\mathrm{H}$ tumor growth in vivo, along with decreased level of p-MYC-Ser62. Taken together, these findings suggest that inhibition of EGFR signal could sensitize HCC cells to JQ1 through attenuating MYC stability in vitro and in vivo. 


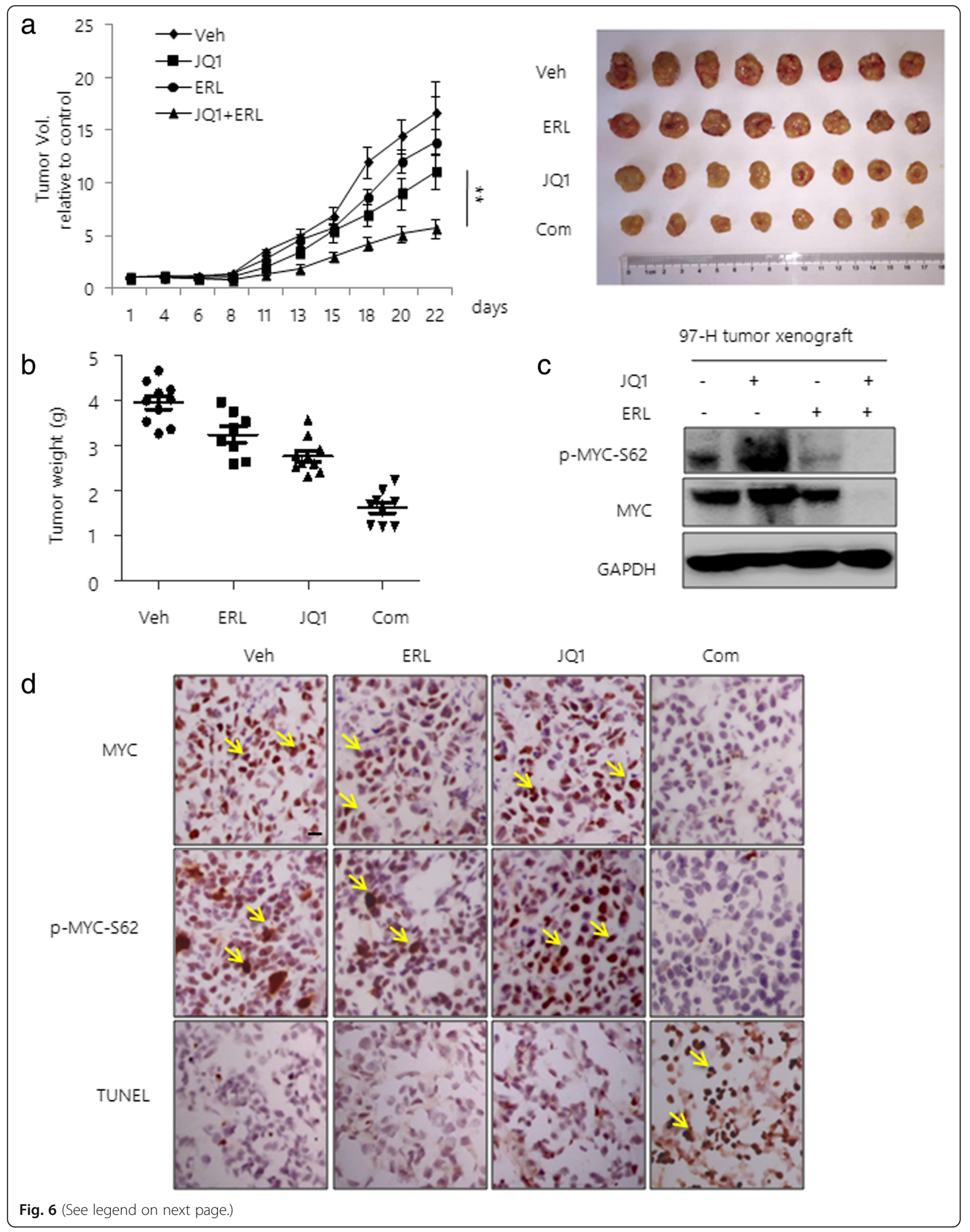


(See figure on previous page.)

Fig. 6 Combination of JQ1 with EGFR inhibitor reduces JQ1 resistant HCC tumor growth. a 97-H cells $\left(5 \times 10^{6}\right)$ were injected into the flanks of CB17/SCID mice. After the subcutaneous tumors reached a size of $10 \mathrm{~cm}^{3}$, mice were treated with vehicle, JQ1 (50 mg/kg), ERL (50 mg/kg) or a combination of the drugs every 2 days. Tumor volumes were monitored during the treatments. Pictures of tumor removed are shown at the right. b Tumor weights are shown after 3 weeks of treatment. $\mathbf{c}$ Western blot analysis of MYC and p-MYC-Ser62 in tumor lysates. GAPDH was used as a loading control. $\mathbf{d}$ Representative immunohistochemistry images. Tumors from mice treated with vehicle, JQ1, ERL or the combination were stained with MYC, p-MYC-Ser62 antibodies and TUNEL. Data are presented as mean \pm s.d. ${ }^{*} p<0.01$, Scale bars, $20 \mu \mathrm{m}$

\section{Conclusion}

In summary, we demonstrated that JQ1 has more potent anti-tumor effects than sorafenib in MYCoverexpressing HCC cells. Furthermore, MAPK activation affects the sensitivity of $97-\mathrm{H}$ cells to JQ1. Mechanistically, we showed that EGFR-I645L, an activating EGFR mutation, impaired the sensitivity of HCC to JQ1 via MAPK regulation of MYC stability. In addtion, combination of JQ1 with EGFR inhibitors significantly enhanced the anti-tumor effect in vitro and in vivo. Since MYC amplification co-occurring with EGFR activation, is frequently observed in advanced HCC (http://www.cbioportal.org), we suggest that EGFR or MAPK status might be considered ahead of JQ1 therapy.

\section{Additional files}

Additional file 1: Figure S1. Prognostic analysis of MYC expression in HCC. (a) Analysis of the risk based on MYC expression in HCC using SurvExpress compilation. (b) The overall survival probability based on MYC expression in HCC patients using the Kaplan-Meier analysis. (TIF 239 $\mathrm{kb})$

Additional file 2: Figure S2. BET inhibitor inhibited tumor growth more potently than sorafenib in MYC-positive HCC cells. HCC cells were treated with either JQ1 or sorafenib for $48 \mathrm{~h}$. Apoptosis was assessed by Annexin V / PI double staining. Quantification of apoptotic cells was determined based on Annexin $\mathrm{V}$ positive cells. (TIF $300 \mathrm{~kb}$ )

Additional file 3: Figure S3. JQ1 resulted in a greater reduction of tumor growth than sorafenib in vivo. (a) BEL-7402 and 97-L cells $\left(5 \times 10^{6}\right.$ each) were injected into the flanks of CB17/SCID mice. After the subcutaneous tumors reached a size of $10 \mathrm{~cm}^{3}$, mice were randomly treated with vehicle, JQ1 or sorafenib at $50 \mathrm{mg} / \mathrm{kg}$ every 2 days. Tumor images is shown. (b) Analysis of apoptosis in 97-L tumor xenografts by $\mathrm{HC}$ staining. Tumors from mice treated with vehicle, JQ1, or sorafenib were stained with H\&E, MYC, and TUNEL. Representative immunohistochemistry images were shown. (c) Immunoblot analysis of tumor lysates treated with vehicle, JQ1 or sorafenib, using the indicated antibodies. (TIF $1170 \mathrm{~kb}$ )

Additional file 4: Figure S4. JQ1 significantly induced apoptosis in MYC-positive HCC cells. HCC cells were treated with JQ1 for $48 \mathrm{~h}$. Apoptosis was assessed by Annexin V / PI double staining. Quantification of apoptotic cells was determined based on Annexin $\mathrm{V}$ positive cells. (TIF $413 \mathrm{~kb})$

Additional file 5: Figure S5. Combination of JQ1 with ERK inhibitor induced cellular apoptosis. HCC cells were treated with JQ1, SCH772984 ( $\mathrm{SCH}$ ) or the combination. Apoptosis was assessed by Annexin V / PI double staining. Quantification of apoptotic cells was determined based on Annexin $V$ positive cells. Representative result of FACS analysis was shown. (TIF $196 \mathrm{~kb}$ )

Additional file 6: Figure S6. Inhibition of EGFR activity overcame the JQ1 resistance. (a) Immunoblot analysis of $97-\mathrm{H}$ cells expressing control shRNA or EGFR shRNA treated with JQ1. (b) HCC cells were treated with
JQ1, Erlotinib (ERL) or the combination. Apoptosis was assessed by Annexin V / PI double staining. Quantification of apoptotic cells was determined based on Annexin $V$ positive cells. Representative result of FACS analysis was shown. (c) Immunoblot analysis of $97-\mathrm{H}$ cells treated with variable doses of JQ1 with or without a fixed dose of ERL. Total lysates were subjected to the indicated antibodies. (TIF $514 \mathrm{~kb}$ )

\section{Abbreviations}

BET: bromodomain and extra-terminal; BRD4: Bromodomain-containing protein 4; DMSO: Dimethyl sulfoxide; ECAR: Extracellular acidification rate; EGFR: epidermal growth factor receptor; ERK: extracellular regulated protein kinases; HCC: hepatocellular carcinoma; MAPK: mitogen-activated protein kinase; PARP: poly ADP-ribose polymerase; PARP-CL: cleaved parp; PARPFL: full parp; qRT-PCR: Quantitative real time polymerase chain reaction; scr: scrambled shRNA; Veh: vehicle; WES: Whole Exome Sequencing; WT: wild type

\section{Acknowledgments}

Not applicable.

\section{Funding}

The study has been supported the National Key Research and Development Program of China (2017YFC1308604), National Natural Science Foundation of China grants (No. 81872446), Innovation program of science and research from the DICP, CAS (DICP TMSR201601), the strategic priority Research of the Chinese Academy of Scienices (SDA16020900), and the 100 Talents Program of Chinese Academy of Sciences.

\section{Availability of data and materials}

The data generated or analyzed during this study are included in this published article and its additional files.

\section{Authors' contributions}

$Y Y$ and $Y L$ designed all the experiments. $Y Y, M S, X Z, C W, P G, X S$ and $Y W$ acquired the data. $Y Y$ and $Y L$ conducted the experiments and analyzed and interpreted the results. $Y L, J Q, S Z$ and $Z Z$ supervised the project. $Y Y$ wrote the draft manuscript. YL and SZ reviewed and edited the manuscript. All authors read and approved the final manuscript.

\section{Ethics approval and consent to participate}

All animal procedures were approved by the Institutional Animal Care and Use Committee of the Dalian Medical University.

\section{Consent for publication}

Not applicable.

\section{Competing interests}

The authors declare that they have no competing interests.

\section{Publisher's Note}

Springer Nature remains neutral with regard to jurisdictional claims in published maps and institutional affiliations.

\section{Author details}

${ }^{1}$ CAS Key Laboratory of Separation Science for Analytical Chemistry, Scientific Research Center for Translational Medicine, Dalian Institute of Chemical Physics, Chinese Academy of Sciences, Dalian 116023, China. ${ }^{2}$ School of Life Science, Dalian University, Dalian 116023, China. ${ }^{3}$ Surgical Neurology Branch, National Institute of Neurological Disorders and Stroke, National Institutes of 
Health, Bethesda, MD 20892, USA. ${ }^{4}$ College of Chemistry and Molecular Sciences, Wuhan University, Wuhan 430072, China. ${ }^{5}$ Department of Hepatobiliary and Pancreatic Surgery, The First Affiliated Hospital of Zhengzhou University, Zhengzhou, Henan, China. ${ }^{6}$ Open and Key Laboratory of Hepatobiliary and Pancreatic Surgery and Digestive Organ Transplantation at Henan Universities, The First Affiliated Hospital of Zhengzhou University, Zhengzhou, Henan, China. 'Zhengzhou Key Laboratory of Hepatobiliary and Pancreatic Diseases and Organ Transplantation, Zhengzhou, Henan, China. ${ }^{8}$ Henan Key Laboratory of Digestive Organ Transplantation, Zhengzhou, Henan, China. 'University of Chinese Academy of Sciences, Beijing 100049, China.

Received: 17 October 2018 Accepted: 6 February 2019 Published online: 15 February 2019

\section{References}

1. Llovet JM, Ricci S, Mazzaferro V, Hilgard P, Gane E, Blanc JF, de Oliveira AC, Santoro A, Raoul JL, Forner A, et al. Sorafenib in advanced hepatocellular carcinoma. N Engl J Med. 2008;359(4):378-90.

2. Cheng AL, Kang YK, Chen Z, Tsao CJ, Qin S, Kim JS, Luo R, Feng J, Ye S, Yang TS, et al. Efficacy and safety of sorafenib in patients in the Asia-Pacific region with advanced hepatocellular carcinoma: a phase II randomised, double-blind, placebo-controlled trial. Lancet Oncol. 2009; 10(1):25-34

3. Chen J, Jin R, Zhao J, Liu J, Ying H, Yan $H$, Zhou S, Liang Y, Huang D, Liang $X$, et al. Potential molecular, cellular and microenvironmental mechanism of sorafenib resistance in hepatocellular carcinoma. Cancer Lett. 2015:367(1):1-11.

4. Guan DX, Shi J, Zhang Y, Zhao JS, Long LY, Chen TW, Zhang EB, Feng YY, Bao WD, Deng $Y Z$, et al. Sorafenib enriches epithelial cell adhesion molecule-positive tumor initiating cells and exacerbates a subtype of hepatocellular carcinoma through TSC2-AKT cascade. Hepatology. 2015; 62(6):1791-803.

5. Negri FV, Dal Bello B, Porta C, Campanini N, Rossi S, Tinelli C, Poggi G, Missale G, Fanello S, Salvagni S, et al. Expression of pERK and VEGFR-2 in advanced hepatocellular carcinoma and resistance to sorafenib treatment. Liver Int. 2015;35(8):2001-8.

6. Abdel-Rahman O, Lamarca A. Development of sorafenib-related side effects in patients diagnosed with advanced hepatocellular carcinoma treated with sorafenib: a systematic-review and meta-analysis of the impact on survival. Expert Rev Gastroenterol Hepatol. 2017;11(1):75-83.

7. Mazzoccoli G, Miele L, Oben J, Grieco A, Vinciguerra M. Biology, epidemiology, clinical aspects of hepatocellular carcinoma and the role of Sorafenib. Curr Drug Targets. 2016;17(7):783-99.

8. Bruix J, Tak WY, Gasbarrini A, Santoro A, Colombo M, Lim HY, Mazzaferro V, Wiest R, Reig M, Wagner A, et al. Regorafenib as second-line therapy for intermediate or advanced hepatocellular carcinoma: multicentre, open-label, phase II safety study. Eur J Cancer. 2013;49(16):3412-9.

9. Kudo M. Systemic therapy for hepatocellular carcinoma: latest advances. Cancers (Basel). 2018;10:11.

10. Bakouny Z, Assi T, El Rassy E, Nasr F. Second-line treatments of advanced hepatocellular carcinoma: systematic review and network meta-analysis of randomized controlled trials. J Clin Gastroenterol. 2018.

11. Zhu AX: Recent developments in systemic therapy for hepatocellular carcinoma. Clin Adv Hematol Oncol 2017, 15 Suppl 9(8):7-13.

12. Inarrairaegui M, Melero I, Sangro B. Immunotherapy of hepatocellular carcinoma: facts and hopes. Clin Cancer Res. 2018;24(7):1518-24.

13. Lin $Y Y$, Tan $C T$, Chen CW, Ou DL, Cheng AL, Hsu C. Immunomodulatory effects of current targeted therapies on hepatocellular carcinoma: implication for the future of immunotherapy. Semin Liver Dis. 2018;38(4): 379-88.

14. Stoelzle T, Schwarb P, Trumpp A, Hynes NE. C-Myc affects mRNA translation, cell proliferation and progenitor cell function in the mammary gland. BMC Biol. 2009;7:63.

15. Lin CY, Loven J, Rahl PB, Paranal RM, Burge CB, Bradner JE, Lee TI, Young RA. Transcriptional amplification in tumor cells with elevated c-Myc. Cell. 2012;151(1):56-67.

16. Dang CV, Resar LM, Emison E, Kim S, Li Q, Prescott JE, Wonsey D, Zeller K. Function of the c-Myc oncogenic transcription factor. Exp Cell Res. 1999. 253(1):63-77.
17. Kawate S, Fukusato T, Ohwada S, Watanuki A, Morishita Y. Amplification of c-myc in hepatocellular carcinoma: correlation with clinicopathologic features, proliferative activity and p53 overexpression. Oncology. 1999;57(2): 157-63.

18. Kato GJ, Dang CV. Function of the c-Myc oncoprotein. FASEB J. 1992;6(12): 3065-72.

19. Lin CP, Liu CR, Lee CN, Chan TS, Liu HE. Targeting c-Myc as a novel approach for hepatocellular carcinoma. World J Hepatol. 2010;2(1):16-20.

20. Patel MC, Debrosse M, Smith M, Dey A, Huynh W, Sarai N, Heightman TD, Tamura T, Ozato K. BRD4 coordinates recruitment of pause release factor $P$ TEFb and the pausing complex NELF/DSIF to regulate transcription elongation of interferon-stimulated genes. Mol Cell Biol. 2013;33(12):2497507.

21. Jung M, Gelato KA, Fernandez-Montalvan A, Siegel S, Haendler B. Targeting BET bromodomains for cancer treatment. Epigenomics. 2015:7(3):487-501.

22. Wang J, Wang Y, Mei H, Yin Z, Geng Y, Zhang T, Wu G, Lin Z. The BET bromodomain inhibitor JQ1 radiosensitizes non-small cell lung cancer cells by upregulating p21. Cancer Lett. 2017;391:141-51.

23. Garcia PL, Miller AL, Kreitzburg KM, Council LN, Gamblin TL, Christein JD, Heslin MJ, Arnoletti JP, Richardson JH, Chen D, et al. The BET bromodomain inhibitor JQ1 suppresses growth of pancreatic ductal adenocarcinoma in patient-derived xenograft models. Oncogene. 2016;35(7):833-45.

24. Filippakopoulos P, Qi J, Picaud S, Shen Y, Smith WB, Fedorov O, Morse EM, Keates T, Hickman T, Felletar I, et al. Selective inhibition of BET bromodomains. Nature. 2010;468(7327):1067-73.

25. Mustafi S, Camarena V, Volmar CH, Huff TC, Sant DW, Brothers SP, Liu ZJ, Wahlestedt C, Wang G. Vitamin C Sensitizes Melanoma to BET Inhibitors. Cancer Res. 2018;78(2):572-83.

26. Kurimchak AM, Shelton C, Duncan KE, Johnson KJ, Brown J, O'Brien S, Gabbasov R, Fink LS, Li Y, Lounsbury N, et al. Resistance to BET Bromodomain inhibitors is mediated by Kinome reprogramming in ovarian Cancer. Cell Rep. 2016;16(5):1273-86.

27. Shimamura T, Chen Z, Soucheray M, Carretero J, Kikuchi E, Tchaicha JH, Gao Y, Cheng KA, Cohoon TJ, Qi J, et al. Efficacy of BET bromodomain inhibition in Kras-mutant non-small cell lung cancer. Clin Cancer Res. 2013;19(22): 6183-92.

28. Lee DH, Qi J, Bradner JE, Said JW, Doan NB, Forscher C, Yang H, Koeffler HP. Synergistic effect of JQ1 and rapamycin for treatment of human osteosarcoma. Int J Cancer. 2015;136(9):2055-64.

29. Ma YF, Wang LH, Neitzel LR, Loganathan SN, Tang N, Qin LL, Crispi EE, Guo Y, Knapp S, Beauchamp RD, et al. The MAPK pathway regulates intrinsic resistance to BET inhibitors in colorectal Cancer. Clin Cancer Res. 2017;23(8): 2027-37.

30. Li GQ, Guo WZ, Zhang Y, Seng JJ, Zhang HP, Ma XX, Zhang G, Li J, Yan B, Tang HW, et al. Suppression of BRD4 inhibits human hepatocellular carcinoma by repressing MYC and enhancing BIM expression. Oncotarget. 2016;7(3):2462-74.

31. Aguirre-Gamboa R, Gomez-Rueda H, Martinez-Ledesma E, Martinez-Torteya A, Chacolla-Huaringa R, Rodriguez-Barrientos A, Tamez-Pena JG, Trevino V. SurvExpress: an online biomarker validation tool and database for cancer gene expression data using survival analysis. PLoS One. 2013;8(9):e74250.

32. Tateishi K, lafrate AJ, Ho Q, Curry WT, Batchelor TT, Flaherty KT, Onozato ML, Lelic N, Sundaram S, Cahill DP, et al. Myc-driven glycolysis is a therapeutic target in glioblastoma. Clin Cancer Res. 2016;22(17):4452-65.

33. Ding SJ, Li Y, Shao XX, Zhou H, Zeng R, Tang ZY, Xia QC. Proteome analysis of hepatocellular carcinoma cell strains, MHCC97-H and MHCC97-L, with different metastasis potentials. Proteomics. 2004;4(4):982-94.

34. Ji S, Qin Y, Shi S, Liu X, Hu H, Zhou H, Gao J, Zhang B, Xu W, Liu J, et al. ERK kinase phosphorylates and destabilizes the tumor suppressor FBW7 in pancreatic cancer. Cell Res. 2015;25(5):561-73.

35. Gonzalez V, Hurley LH. The c-MYC NHE III(1): function and regulation. Annu Rev Pharmacol Toxicol. 2010;50:111-29.

36. Delmore JE, Issa GC, Lemieux ME, Rahl PB, Shi J, Jacobs HM, Kastritis E, Gilpatrick T, Paranal RM, Qi J, et al. BET bromodomain inhibition as a therapeutic strategy to target c-Myc. Cell. 2011;146(6):904-17.

37. Qiu HF, Jackson AL, Kilgore JE, Zhong Y, Chang LLY, Gehrig PA, Zhou CX, Bae-Jump VL. JQ1 suppresses tumor growth through downregulating LDHA in ovarian cancer. Oncotarget. 2015;6(9):6915-30. 